\title{
ALGUNAS CONSIDERACIONES SOBRE LAS HUMANIDADES DESDE LA ERA DIGITAL ${ }^{1}$
}

\author{
Some Considerations on Humanities from the Digital Age
}

\author{
Teresa Ayala Pérez*
}

\begin{abstract}
RESUMEN
Referirse a las humanidades en el siglo XXI no es novedoso si se considera que estas han sido objeto de estudio durante siglos. No obstante lo anterior, reflexionar respecto de las humanidades desde la era digital, cuando pareciera que no tienen lugar, sí resulta pertinente. En la sociedad de la información las ciencias exactas se adecuan perfectamente a este paradigma, sin embargo, las humanidades parecieran alejarse de él. El presente trabajo intenta destacar la importancia de las humanidades, abordándolas desde los studia humanitatis hasta las humanidades digitales, con especial atención a la dicotomía ciencia-humanidades y a la presencia de las humanidades en el discurso académico.
\end{abstract}

Palabras clave: humanidades, ciencia, las Dos Culturas, humanidades digitales, discurso académico.

${ }^{1}$ El presente trabajo se origina en el proyecto de investigación "Cibercultura y formación inicial docente en carreras pedagógicas del área de humanidades” (DIUMCE FGI 04-15).

* Departamento de Castellano, Universidad Metropolitana de Ciencias de la Educación. Santiago, Chile. Correo electrónico: teresa.ayala@umce.cl

Artículo recibido el 9 de agosto de 2018. Aceptado el 7 de noviembre de 2018. 


\begin{abstract}
It is not new to referring to the humanities in the 21 st century because these have been studied for centuries. However, reflecting on the humanities from the digital age, when it seems that they do not take place, is relevant. In the information society the exact sciences are perfectly suited to this paradigm, but the humanities seem to move away from it. The present work tries to emphasize the importance of the humanities, approaching them from the studia humanitatis to the digital humanities, with special attention to the dichotomy science-humanities and the humanities presence in the academic discourse.
\end{abstract}

Keywords: humanities, cyberculture, science, the Two Cultures, digital humanities, academic discourse.

Science is what you know, philosophy is what you don't know (Bertrand Russel)

\title{
1. Introducción
}

Las últimas décadas han estado marcadas por la presencia de la tecnología digital y, en este contexto, cabe preguntarse, entonces qué papel y cuál es el lugar de las humanidades dentro de una sociedad dependiente de dicha tecnología, donde además es el discurso de las ciencias naturales el que domina la producción académica. Por estos motivos, el presente trabajo intenta mostrar distintos enfoques respecto del concepto de humanidades desde su origen hasta las humanidades digitales, destacar su vigencia e importancia, su relación con la ciencia y su lugar dentro del actual paradigma cultural, especialmente en el ámbito de las publicaciones académicas.

\section{Humanidades}

Según Morón Arroyo (2002) las humanidades, en sentido estricto, son el estudio de las disciplinas que constituyen la llamada "alta cultura" que incluye artes, teología, literatura, filosofía, pero no son y nunca deben pretender 
ser ciencias, aunque deben ser "conocimiento riguroso" 2 . Las humanidades - de acuerdo con la tradición- han sido concebidas a partir de una "cultura común" (occidental, clasista y jerárquica), basada en el dominio de la cultura "clásica". Por este motivo, la especialización emanaba "no del dominio de habilidades, técnicas y procedimientos, como en las ciencias naturales, sino de un habitus particular" (López-Bonilla, 2013: 394). Este habitus implica un especialista en la disciplina, un conocedor ideal, lo que conlleva la idea de que las humanidades se encuentran alejadas del acervo cotidiano de los integrantes de la sociedad, al mismo tiempo que se oponen a un tipo de conocimiento útil y práctico.

Como afirma Proctor (1998), la expresión studia humanitatis ("afán de la cultura" de acuerdo con Reeve ${ }^{3}$, 1996) es acuñada a partir de los escritos de Cicerón (Pro Murena 61 y Pro Caelio 24). Los humanistas del Renacimiento usaron la frase para nombrar su programa de educación a partir de una oración del discurso de Cicerón Pro Archia, elaborado como defensa del poeta Licino Archías, manuscrito descubierto por Petrarca en 1333. Cicerón afirma que “[...] finalmente, presidiendo este pretor ${ }^{4}$ el juicio, me permitáis hablar un poco más libremente de los estudios de humanidades y de las letras [...]”. Pro Archia es el primer texto destinado centralmente a llamar la atención sobre la importancia personal y social de las letras, de la actividad literaria y de los estudios literarios (Arbea, 2002: 393). Proctor afirma que en Cicerón studia humanitatis es otro nombre para las artes, artes humanitatis, pues ambos nombres son intercambiables en sus escritos, quien entiende que son todas aquellas artes relacionadas que se estudian por primera vez en la juventud y que ayudarán a alcanzar la humanitas. Para Morón Arroyo (2002), Cicerón

\footnotetext{
2 "Humanities in the strict sense are language, literature, philosophy, and other disciplines that constitute the so-called «high culture». [...] High culture includes art, theology, literature, philosophy [...] The humanities are not and should never pretend to be sciences, but they must be «rigorous knowledge» (strenge Wissenschaft), a Husserl intimated for philosophy in his programmatic study of 1911" (Morón Arroyo, 2002: 4 y ss.).

${ }^{3}$ Se refiere a Michael D. Reeve, 1996, "Classical Scholarship", en J. Kraye (ed.) The Cambridge Companion to Renaissance Humanism. Cambridge University Press, pp. 20-46. 4 "Magistrado romano que ejercía jurisdicción en Roma o en las provincias" (DLE, $23^{\mathrm{a}}$ Ed.).
} 
entendía humanitas como el ideal al cual todos los estudios o la educación aspira: gramática y retórica, leyes, historia romana, astronomía. De acuerdo con Mann (1998), en el lenguaje académico de la Italia cuatrocentista la voz umanista devino habitual para referirse a quien enseñara o estudiara la literatura clásica y las disciplinas que la acompañaban. Cordua afirma que [...] "umanista", fue usado primero para designar a los profesores de retórica de algunas universidades italianas y es vertida del vernáculo al latín" (Cordua, 2013: 9). Cordua (2012) sostiene que cuando hoy hablamos de "humanidades" no hay referencia a la concepción renacentista, cuestionada y abandonada el siglo pasado, sino que

Usamos esta designación, en cambio, para un grupo de disciplinas que formaban parte de todos los niveles de la educación formal que se impartía todavía hace algunas décadas. Estas disciplinas comprenden a la filosofía, la historia, la lingüística, las ciencias sociales y políticas, las artes y la literatura, el derecho, ciertas variantes de la sicología y la antropología y algunos aspectos de las ciencias estrictas y las especiales de la naturaleza (Cordua, 2012: 7).

Heidegger $(1946)^{5}$ le responde al filósofo Beaufret quien se pregunta “¿comment redonner un sens au mot «Humanisme»?";

La humanitas es pensada por vez primera bajo este nombre expreso y se convierte en una aspiración en la época de la república romana. El homo humanus se opone al homo barbarus. El homo humanus es ahora el romano, que eleva y ennoblece la virtus romana al "incorporarle" la $\pi \alpha \imath \delta \varepsilon ́ ı \alpha$ tomada en préstamo de los griegos. Estos griegos son los de la Grecia tardía, cuya cultura era enseñada en las escuelas filosóficas y consistía en la eruditio e institutio in bonas artes. La $\pi \alpha 1 \delta \varepsilon ́ 1 \alpha$ así entendida se traduce mediante el término "humanitas". La auténtica romanitas del homo humanus consiste precisamente en semejante humanitas. En Roma nos encontramos con el primer humanismo. Y, por eso, se trata en su esencia de un fenómeno específicamente romano que nace del encuentro de la romanidad con la cultura de la Grecia tardía. El que se conoce como Renacimiento de los siglos XIV y XV en Italia es una renascentia romanitatis. Desde el momento en que lo que le

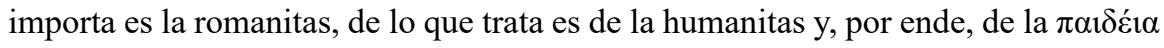
griega" (Heidegger, 2010: 22).

Small (2013) afirma que, desde un nivel general, las humanidades

\footnotetext{
5 Brief über den Humanismus, 'Carta sobre el humanismo'.

¿CCómo dar sentido a la palabra "humanismo"? (Trad. de la autora)
} 
estudian las prácticas de creación de significado de las culturas humanas, pasadas y presentes, centrándose en la interpretación y la evaluación crítica, principalmente en términos de la respuesta individual y con un elemento ineliminable de subjetividad ${ }^{7}$; pero esta descripción inicial no proporciona una valoración, sino más bien una especificación básica de los objetos de estudio y modos de conocimiento sobre la base de los cuales se pueden hacer afirmaciones de valoración.

\section{Ciencia y humanidades}

En 1959 Charles Percy Snow dictó la célebre conferencia en la Universidad de Cambridge The Two Cultures and the Scientific Revolution ${ }^{8}$ que dio origen al libro del mismo nombre y que, a su vez, ampliaba un artículo suyo publicado en 1956: "The Two Cultures". En dicha conferencia presentaba la tesis de que existen dos culturas que se originan en una incomunicación entre las humanidades (los humanistas) y la ciencia (los científicos-tecnólogos). Snow plantea que cada grupo tiene una imagen distorsionada del otro, pero que

El punto de choque de dos materias, dos disciplinas - dos galaxias, según van las cosas- debería producir oportunidades creativas. Las ha habido en la historia de la actividad mental, y de ellas surgieron algunos de los adelantos decisivos. Hoy existen las posibilidades. Pero existen, por decirlo así, en un vacío, porque los integrantes de las dos cultural no saben hablar unos con otros (Snow, 2000: 86).

La postura de Snow ha sido criticada, asunto que él mismo asume en la "segunda mirada" respecto de las dos culturas, sin embargo, su análisis puede ser considerado igualmente válido por cuanto hasta hoy no se observan líneas de investigación que hagan dialogar a estas dos culturas. En 1962, también en Cambridge, en la lectura “Two cultures?”, el crítico Frank Leavis defendía la cultura literaria frente a la científica, insistiendo en que la mera adquisición de conocimientos no significa realmente cultura por cuanto esta debía ser un enriquecimiento del espíritu. En 1995, John Brockman publica The Third

\footnotetext{
7 "The humanities study the meaning-making practices of human cultures, past and present, focusing on interpretation and critical evaluation, primarily in terms of the individual response and with an ineliminable element of subjectivity" (Small, 2013: 23).

${ }^{8}$ Las dos culturas contiene la conferencia de 1959 y una segunda parte que titula "Las dos culturas II. Una segunda mirada" (1963).
} 
Culture, retomando las ideas de Snow respecto de la separación entre la cultura humanística y la científica. Esta tercera cultura sería la superación de la tradicional falta de comunicación, aspecto que para Brockman es fundamental, pues considera que el quehacer del intelectual incluye la comunicación con el público y la ciencia se convierte en "cultura pública". Esta tercera cultura acerca la ciencia a las personas y refleja el creciente interés social por la actividad científica, lo cual constituye un nuevo humanismo, una hibridación entre ciencias y letras. Para Llopis Goig no sería efectivo que existe un acercamiento entre ciencia y humanidades por cuanto "la conciliación entre las dos culturas no se puede circunscribir a que los científicos se comuniquen con los grandes públicos, pues la conciliación entre las dos culturas no se puede ceñir a divulgar ciencia" (Llopis Goig, 2008: 172).

Jerome Kagan publica en 2009 la obra The Three Cultures. Natural Sciences, Social Sciences, and the Humanities in the 21st Century, cuyo subtítulo es Revisiting C. P. Snow. Postula que ha surgido una tercera cultura, pero a diferencia de Brockman, se refiere a las ciencias sociales: sociología, psicología, antropología, economía y ciencia política. Afirma que algo tienen en común las tres culturas: que sus afirmaciones científicas solo son válidas y coherentes dentro de su propia comunidad científica. Kagan muestra su preocupación por la vida intelectual occidental y la importancia que adquieren las ciencias naturales después de la Segunda Guerra Mundial, lo que habría 'intimidado' a las otras dos comunidades académicas, pues existiría cierta obsesión con una visión biológicamente materialista de la condición humana. Kagan no rebate ni defiende las afirmaciones de Snow, pero sí mantiene la visión de la falta de comunicación entre las distintas culturas científicas que, de hecho, se ha mantenido o ha crecido desde la conferencia de Snow e incluso parecen haberse agravado en el transcurso de los últimos años según Sokal y Bricmont ${ }^{9}$ (1997). Kagan agrega que existen nueve dimensiones que diferencian a las ciencias naturales, las ciencias sociales y las humanidades:

\footnotetext{
${ }^{9}$ Autores que ridiculizaron a una prestigiosa revista académica a través de un texto que parodiaba el lenguaje filosófico postmodernista y luego publicaron el libro Fashionable Nonsense: Postmodern Intellectuals. Abuse of Science.
} 
principales preocupaciones, fuentes de evidencia, vocabulario utilizado, importancia de las condiciones sociales, importancia de los valores éticos, dependencia del financiamiento externo, condiciones de trabajo, contribución a la economía nacional y los criterios cada grupo cuando juzgan un cuerpo de trabajo como elegante o hermoso ${ }^{10}$. En todo caso, Kagan afirma que "It is time for the members of the three cultures to adopt a posture of greater humility" (Kagan, 2009: 275). Gould (2003), en tanto, utiliza la metáfora de las formas de sobrevivencia del zorro y el erizo ${ }^{11}$ en una obra que intenta analizar las relaciones entre ciencia y humanidades. En el Prefacio sostiene que

Utilizo el zorro y el erizo para mi modelo por la manera en que las ciencias y las humanidades deben interactuar, porque creo que ninguna estrategia pura puede funcionar, pero que una unión fructífera de estos opuestos y aparentemente tan distantes puede articularse, con buena voluntad y una moderación importante por ambos bandos, en una empresa diversa pero común de unidad y poder. La manera del erizo no puede bastar porque las ciencias y las humanidades, pero la lógica básica de sus esfuerzos dispares, realizan cosas distintas, cada una de ellas igualmente esencial para la humanidad (Gould, 2010: 7).

Rens Bod (2013) se pregunta ¿Es el famoso debate de dos culturas que fue desencadenado por C.P. Snow en 1959 solo un fenómeno de los últimos cincuenta años o ha existido antes? ¿Las ideas y descubrimientos en las humanidades alguna vez han conducido a avances 'científicos'? Para Bod, desde el siglo XIX han sido definidas como disciplinas que investigan las expresiones de la mente humana, como lenguaje, arte, música o poesía y el estudio de los seres humanos en su contexto social constituyen las ciencias sociales (como sociología, psicología, antropología y economía). Estas definiciones resultarían insatisfactorias, pues la matemática es producto de la mente humana, pero no se considera una disciplina humanista. Bod aclara que

\footnotetext{
10 "The criteria members of each group use when they judge a body of work as elegant and beautiful".

${ }^{11}$ De acuerdo con Beorlegui (2016), Berlin toma la metáfora del zorro y el erizo de un proverbio del poeta griego Arquíloco: "Mientras que el zorro sabe muchas cosas, el erizo sabe mucho de una sola cosa", considerando más positiva la postura del erizo que la del zorro. Esta idea, agrega Beorlegui, posteriormente fue recogida en la fábula de Esopo "El zorro y el gato", donde la actitud del erizo es sustituida por la del gato, con el mismo criterio de superioridad de la actitud del gato respecto a la del zorro.
} 
el término 'humanidades' es ambiguo en el mundo anglófono, pues no solo se refiere a una rama de las disciplinas académicas, sino que también puede ser usado para aludir a los temas estudiados, por lo cual los dos significados pueden confundirse. Por este motivo, Bod sostiene que él usa el término humanidades para referirse a los estudios de literatura, música, arte, teatro, etc., que corresponde al alemán Geiteswissenchaften ('ciencias del espíritu'), al italiano umanistiche ('ciencias humanas') o al holandés alfawetenschappen ('ciencias alfa'). Asimismo, en el mundo anglófono se utiliza la sigla $S S H$, Social Sciences and Humanities que, de acuerdo con Reale, Avramom et.al. (2017), poseen sus propias características epistemológicas y sus resultados las diferencian de las disciplinas de ciencia, tecnología, ingeniería y matemáticas (STEM). Para De Zubiría (1990), y como evolución fundamental, el término humanidades fue perdiendo fuerza como referencia al estudio de las lenguas y letras clásicas y se afirmó, en cambio, "como vocablo para designar el cultivo del mundo interior del hombre. Surgió, entonces, la expresión de ciencias humanas, con la cual también se las nombra, para diferenciarlas de las ciencias naturales, las que estudian la naturaleza, o sea, el mundo exterior al hombre" (Zubiría, 1990: 490).

\section{Las humanidades hoy}

En una sociedad donde aparentemente lo más importante es la productividad y el éxito profesional, los graduados de estudios humanísticos parecieran no tener lugar en el mercado laboral, pero -quizás por el mismo motivo- estos estudios pueden resultar más interesantes y desafiantes, pues, de hecho, resulta prácticamente imposible abordar el estudio de muchos temas actuales, por ejemplo la cibercultura, sin recurrir, de una u otra forma, a filósofos, historiadores o estudiosos de la cultura o del lenguaje. Pero la educación formal, en cualquiera de sus niveles y por distintas razones, no está fortaleciendo a las humanidades, sino que, al parecer, las está debilitando. Profesores e intelectuales se lamentan en cartas a periódicos, columnas de opinión o artículos académicos respecto de que ni en las escuelas ni en las 
universidades se enseña latín o griego, que la ortografía y la redacción de los jóvenes es cada vez más precaria o que la comprensión lectora presenta índices extremadamente bajos; sin embargo, casi no se comenta que, en la mayoría de los casos, las autoridades educacionales a través de ministerios, secretarías o agencias exigen que las mallas curriculares contengan cada vez menos asignaturas y que la titulación sea lo más temprana posible, privilegiando, además, aquellas asignaturas más “prácticas”. Al respecto, Nussbaum (2010) sostiene que

\begin{abstract}
en casi todas las naciones del mundo se están erradicando las materias y las carreras relacionadas con las artes y las humanidades, tanto a nivel primario y secundario como a nivel terciario y universitario. Concebidas como ornamentos inútiles por quienes definen las políticas estatales en un momento en que las naciones deben eliminar todo lo que no tenga ninguna utilidad para ser competitivas en el mercado global, estas carreras y materias pierden terreno a gran velocidad, tanto en los programas curriculares como en la mente y el corazón de padres e hijos (Nussbaum, 2010: 20).
\end{abstract}

De acuerdo con Hutner y Mohamed (2016), el concepto de "crisis" de las humanidades es autodestructivo, pues si en el lenguaje administrativo crisis es "oportunidad", no es difícil adivinar que se relaciona con la caída de las matrículas y las medidas de austeridad diseñadas para eliminar programas considerados como un obstáculo para los recursos institucionales. La "crisis" puede permitir el cierre de un programa y la "reestructuración" como consigna para terminar o mezclar programas. No obstante lo anterior, es indudable que los cambios sociales y culturales que comenzaron a desarrollarse en el siglo XX contribuyeron al lugar que hoy ocupan las humanidades, por lo que no puede desconocerse la necesaria la evolución y no puede compararse un período histórico con otro. Bérubé (2017) del Consortium of Humanities Centers and Institutes ${ }^{12}$ sostiene que en los últimos años ha llegado a creer que la vulnerabilidad estructural de las humanidades es de tal duración que ya no puede usar el término "crisis", como solía hacerlo en el pasado. Esta situación se ve exacerbada por el hecho de que no existe una base ampliamente accesible o aceptada para la investigación en humanidades; pocas personas

${ }^{12} \mathrm{https} / / /$ chcinetwork.org/ideas/the-humanities-and-the-advancement-of-knowledge. 
ajenas a la academia -y muy pocos administrativos dentro de ella-pueden dar una explicación concisa y convincente de por qué es necesaria una nueva investigación en humanidades. Sin embargo, el porqué se necesitan nuevos modos de interpretación de las humanidades no se explica por sí mismo, por cuanto las ciencias pueden producir nuevos conocimientos (y por eso son ampliamente aceptadas), pero no producen sabiduría. Así, los argumentos a favor de las humanidades proliferan, mientras que los argumentos sobre el valor de la investigación en humanidades siguen siendo escasos.

Por otra parte, Gretchen Busl (2015) ${ }^{13}$ destacaba que las humanidades están experimentando una crisis: el apoyo público ha disminuido, la inscripción de carreras humanísticas se ha reducido y los cursos están desapareciendo de los planes de estudios universitarios, pero parte del problema tiene que ver con el impacto de la investigación en humanidades. La mayoría de los argumentos para "salvar" las humanidades se centran en el supuesto de que los empleadores valoran el pensamiento crítico y las habilidades de comunicación que desarrollan los estudiantes de pregrado, que puede ser cierto, pero con ello se resalta el valor del estudio en el aula, pero no el valor de la investigación. Pero la propia academia, según Busl, es en parte culpable del problema por cuanto se ha hecho poco esfuerzo por demostrar qué propósito puede tener el trabajo en humanidades más allá de la sala de conferencias o de la revista académica. Por el contrario, los estudiosos de las humanidades tienen la expectativa de producir artículos revisados por pares y monografías publicadas por editoriales universitarias para su promoción, lo cual es un sistema anticuado que alienta a los académicos a escribir y hablar solo para una audiencia de pares. Sin embargo, las aplicaciones de este tipo de investigación son enormes, por ejemplo, en organizaciones gubernamentales y $\sin$ fines de lucro. El proyecto Humanities Indicators $^{14}$ de la American Academy of Arts \& Sciences tiene como propósito entregar datos estadísticos a distintas organizaciones como universidades, fundaciones o bibliotecas sobre

\footnotetext{
${ }^{13}$ En el diario británico The Guardian. Ver https://www.theguardian.com/higher-educationnetwork/2015/oct/19/humanities-research-is-groundbreaking-life-changing-and-ignored.

${ }^{14} \mathrm{https}: / / \mathrm{www} . h u m a n i t i e s i n d i c a t o r s . o r g /$
} 
la fuerza laboral en humanidades, fuentes de financiamiento de programas, impacto de las humanidades, etc. Este tipo de iniciativas no solo hace visibles las investigaciones, sino que también intenta establecer vínculos con el resto de la sociedad. Por otra parte, es importante recalcar que las humanidades y las ciencias sociales (SSH) sí tienen valor fuera de la academia, tal como lo muestra la organización Horizon 20, The EU Framework Programme for Research and Communication de la Comisión Europea ${ }^{15}$, que muestra ejemplos del rol de las SSH: salud, cambio demográfico, cambio cultural, acción climática, eficiencia de recursos, transporte verde e integrado, porque la investigación SSH analiza aspectos socioeconómicos, estudios prospectivos y prospectiva tecnológica. Agrega que el liderazgo en tecnologías habilitadoras e industriales, las artes y las humanidades podría ser una fuente esencial para la creatividad en el desarrollo de servicios y diseño de productos. $\mathrm{Al}$ respecto, cabe recordar que en los años 30 Franklin Delano Roosevelt fue asesorado por economistas, abogados y académicos de renombre para combatir la Gran Depresión, cuyo aporte se conoce como brain trust $\mathrm{y}$, en este mismo ámbito, se encuentran los llamados public intellectuals ${ }^{16}$, personajes reconocidos que se mueven entre la literatura y la política, como Tom Wolfe ${ }^{17}$ o Samuel Huntington ${ }^{18}$, cuyas opiniones pueden influenciar a la opinión pública.

\subsection{El discurso académico: ciencia y humanidades}

Por otra parte, en las últimas décadas la producción académica se ha vuelto ineludible para quienes laboran en instituciones de educación superior, pero, según Kagan (2009), las tres culturas (ciencias, ciencias sociales y humanidades) representan comunidades lingüísticas que imponen redes de significado distintos y poco transparentes sobre sus conceptos importantes,

\footnotetext{
${ }^{15} \mathrm{https}$ ://ec.europa.eu/programmes/horizon2020/en/area/social-sciences-humanities

${ }^{16}$ De acuerdo con el Oxford English Dictionary, alude a "....n intellectual who expresses views (especially on popular topics) intended to be accessible to a general audience". Su fuente se encontraría en el New York Times en los años '60. Ver https://en.oxforddictionaries. com/definition/public_intellectual

${ }^{17}$ Tom Wolfe (1930-2018), escritor y periodista estadounidense.

${ }^{18}$ Samuel Huntington (1927-2008), cientista político, autor de The clash of civilizations and the remaking of world order (1996).
} 
pues los vocabularios de cada cultura contienen conceptos con definiciones técnicas que son de interés principal solo para un grupo. Asimismo, se sugiere (o se exige) que los artículos sean enviados a revistas indexadas, especialmente a las incluidas en las plataformas o bases de datos más prestigiosos, pero son las revistas de ciencias la que tienen mayores índices de impacto y las de humanidades quedan relegadas a un segundo término. Esto, junto a que se otorgan más fondos a investigación a ciencias que a humanidades, trae como consecuencia que la producción de artículos académicos en esta área sea menor y deba adecuarse al modelo que imponen las ciencias exactas, por ende, los tradicionales ensayos tienen escasa o nula cabida dentro de las revistas científicas, por cuanto estas privilegian los artículos que dan cuenta de un proyecto de investigación y ojalá muestre resultados empíricos cualitativos o cuantitativos. Así, lo que podría haber sido una reflexión que contribuye al saber y que eventualmente puede motivar otros trabajos, termina siendo solo un artículo con datos estadísticos que resultan adecuados para las ciencias, pero no necesariamente para las humanidades, aunque se debe destacar que en el caso de las ciencias sociales, es posible compatibilizar los dos enfoques. De acuerdo con Rodríguez-Yunta (2017)

Actualmente los indicadores extraídos de Web of Science o Scopus resultan inaplicables para las humanidades. El mayor problema radica en las claras diferencias de datos que pueden obtenerse para cada disciplina, distorsionando la posición de las revistas según donde se clasifiquen y perjudicando a las comunidades investigadoras de menor tamaño, o con diferentes hábitos de citación (RodríguezYunta, 2017: 234).

De acuerdo con Thelwall y Delgado (2015), las monografías son particularmente importantes en humanidades, pero las citas de libros son difíciles de encontrar, porque los índices actuales están dominados por revistas académicas. También existen problemas de cobertura internacional que afectan más a las humanidades que a las ciencias, pero tienen aun menos presencia las conferencias referidas a la investigación de las artes y humanidades. Asimismo, mientras los científicos y científicos sociales pueden afirmar que están construyendo una estructura jerárquica de conocimiento 
en la que es importante citar trabajos previos para demostrar la posición del nuevo trabajo, esto no es así en artes y humanidades; en las artes se valora la creatividad, hasta cierto punto lo opuesto a la construcción jerárquica del conocimiento, y los especialistas en humanidades pueden citar para demostrar la originalidad de su trabajo en lugar de mostrar su contribución al cuerpo de conocimiento. En algunos campos necesitan citar fuentes primarias antiguas, pero esto puede tender a suprimir las citas de la investigación contemporánea. En ciencias, los temas candentes pueden ser los más citados; en humanidades, la investigación en áreas de moda puede mirarse con cierta sospecha y, por lo tanto, altos recuentos de citas reflejan lo opuesto a la opinión de la comunidad en general, probablemente porque los especialistas en humanidades son más especializados y menos proclives a contribuir en equipos que los científicos. Según los autores, es probable entonces que el recuento de citas sea, en el mejor de los casos, un indicador débil del impacto académico en las artes y las humanidades. Desde nuestra perspectiva, uno de los problemas que afecta al discurso académico de las humanidades es la exigencia de privilegiar publicaciones de los últimos cinco años, situación comprensible en el caso de las ciencias, pero no determinante en humanidades. Un paper que da cuenta de avances científicos corre el riesgo de no tener validez al poco tiempo, pues nuevos avances permiten un veloz desarrollo de cada disciplina. Como afirma Santos, el paper es "un escrito fundamentalmente efímero y su contenido, por lo tanto, también lo es" (Santos, 2012: 205). Las disciplinas de humanidades también evolucionan, pero el proceso es distinto, con otros objetivos, por lo tanto, si se aplica de manera inflexible el paradigma de las ciencias "duras", sería imposible citar a Cicerón o Heidegger, lo cual refleja la rigidez del enfoque, porque el hecho de mencionar a clásicos junto con autores actuales muestra la reflexión propia de las humanidades, pues permite complementar las ideas más tradicionales con las vigentes y, de esta forma, observar cómo evolucionan conceptos y perspectivas.

McLeod, Broady-Preston y Atkins (2014) afirman que la presión sobre las humanidades es grande a partir de las métricas impuestas aplicadas por las instituciones y los ejercicios de financiación gubernamentales. De acuerdo 
con Belfiore y Upchurch (2013), el valor que la investigación de las artes y las humanidades otorga a la sociedad no siempre es tan transparente o visible como para otros tipos de investigación, como las innovaciones médicas o tecnológicas. Como resultado, hay un consenso público menor acerca de los beneficios de la investigación de las artes y las humanidades en general, pero es difícil demostrar el impacto socioeconómico de algunas becas de arte y humanidades, a pesar de ser valiosas de otras maneras, algunas de las cuales son imposibles de medir o incluso estimar de manera significativa. Para Kaplan (2017), los profesores de humanidades y ciencias sociales ahora se evalúan de acuerdo con criterios generalmente desarrollados para ciencias duras o de acuerdo con métricas que no captan por completo el impacto de la erudición, es decir, el número de citas que obtiene una pieza de escritura, por ejemplo. Sin embargo, una frase atormenta a los académicos, publish or perish 'publicar o perecer', la cual se utiliza para referirse a una actitud o práctica dentro de las instituciones académicas, por la cual los investigadores están bajo presión para publicar material con el fin de conservar sus puestos. Imad A. Moosa (2018) publica Publish or Perish: Perceived Benefits Versus Unintended Consequences donde se centra en cómo las vidas académicas, la investigación y la enseñanza han sido, sino destruidas, al menos rotas en los últimos veinte años por esta práctica. Asimismo, usar los factores de impacto en los comités de selección y promoción para distinguir entre los registros de publicación de los académicos es un procedimiento dudoso, especialmente cuando se comparan los registros de publicación en diferentes disciplinas. Moosa afirma que-según Geroni y Haddow (2009)- si bien las citas proporcionan una buena medida de la calidad de las revistas científicas, son mucho menos útiles para clasificar las revista de humanidades, "hasta el punto de ser casi inútiles", por lo tanto, no se puede calcular un factor de impacto significativo en estos casos. Por otra parte, HuMetricsHSS ${ }^{19}$ es una iniciativa para repensar los indicadores en humanidades y ciencias sociales que se esfuerza por crear y apoyar un marco basado en valores para comprender y evaluar todos los aspectos de la vida

\footnotetext{
${ }^{19} \mathrm{https}: /$ humetricshss.org/
} 
académica y no solo los datos bibliométricos. Asimismo, en la "Declaración sobre los índices de citación y las prácticas editoriales"20 (2015) presentada por los editores de más de cien revistas académicas latinoamericanas se afirma que las humanidades fortalecen y alientan la apropiación crítica de la cultura y la tradición, abren espacios de discusión y debate, y tienen una dimensión utópica que va más allá de la mera solución de problemas inmediatos. Por eso, las humanidades no se adaptan fácilmente a los criterios meramente cuantitativos, ni a las formas estandarizadas de producción académica.

La World Humanities Conference (2017) organizada por la Unesco y la CIPSH (International Council for Philosophy and Humanistic Studies), por su parte, presentó su documento final titulado "Una nueva agenda de las humanidades para el siglo XXI"21, donde se explica que se debe reconocer que las humanidades tienen una competencia y responsabilidad específicas para fomentar la libertad, la diversidad de pensamiento y la transparencia, fundamentales para todos los aspectos de la vida en la sociedad, mientras enfatizan el papel insustituible de las humanidades para un enfoque crítico de los valores y para la comprensión de procesos a largo plazo, como los desafíos relacionados con los cambios ambientales y las migraciones globales. Asimismo, se insta a establecer mecanismos transparentes de financiamiento internacional colaborativo para las humanidades, asegurando la independencia académica de los investigadores y una mayor difusión de los nuevos conocimientos resultantes de tales esfuerzos; establecer mecanismos en cada país para sintetizar los resultados de los estudios de humanidades y presentarlos a los responsables de la formulación de políticas y la sociedad civil; fomentar la investigación disciplinaria e interdisciplinaria con otros ámbitos de la investigación científica y humanística, así como con las artes, las letras y el conocimiento no académico, mientras también colaboran en la concepción de nuevas formas de enseñanza de las humanidades en educación

\footnotetext{
20 "Declaración sobre los índices de citación y las prácticas editoriales". Innovar: Revista de ciencias administrativas y sociales, Vol. 26, No. 59 (Enero-marzo 2016), pp. 180-184.s/. En https://www.jstor.org/stable/43786578?seq=1\#page_scan_tab_contents.

${ }^{21}$ Ver: http://www.humanities2017.org/en/content/new-humanities-agenda-21st-century.
} 
general y para el aprendizaje de por vida. Lo anterior es una muestra de que en distintos ámbitos, las humanidades intentan mostrar su valor y evidenciar que su presencia también es importante para la sociedad toda y no solo para los claustros académicos.

\section{Cibercultura y humanidades digitales}

Así como la palabra humanidades se relaciona con la "alta cultura", la palabra tecnología se relaciona actualmente con la informática. La tecnología digital ha generado la llamada cibercultura (Castells, 1996; Dery, 1997; De Kerckhove, 1997; Tofts y McKeich, 1998; Lévy, 2001; Ardèvol, 2002; Piscitelli, 2002; Scolari, 2008) la que, para Ardèvol (2002), "no es un nuevo modelo cultural, sino que indica una serie de prácticas sociales significativas". En esencia, se trata de una forma de vida que involucra prácticamente todas las actividades del individuo, se caracteriza por la interactividad, la virtualidad y la velocidad, es decir, aparentemente lo contrario de la reflexión propia de las humanidades. No obstante lo anterior, la tecnología digital ha permitido poner al alcance de millones de personas un número incalculable de creaciones culturales propias de las humanidades, tales como grandes obras literarias, filosóficas o teológicas; recorridos virtuales a museos y sitios patrimoniales, incluso de lugares que ya no existen, o acceso a grandes obras musicales, por citar solo algunos casos. Sin embargo, para algunos, el actual entorno cultural, claramente dominado por la informática, constituye una amenaza a las humanidades. Como afirma Reynal (1998), por haber depositado plenamente su confianza en los instrumentos, ha caído en el anonimato y "se ha deshumanizado" (Reynal, 1998: 13). No obstante, hoy en día las humanidades requieren de la tecnología, en tanto que esta última puede ser enriquecida por las humanidades. Frente a los temores respecto al debilitamiento de estas disciplinas, es posible afirmar que siguen creciendo en todo lugar donde se busque y desarrolle el saber.

Desde otra perspectiva, Derrida $(1998)^{22}$ proponía hablar de las nuevas

\footnotetext{
${ }^{22}$ Conferencia dictada en la Universidad de Standford en 1998 y, en 2001, en la Universidad de Murcia.
} 
humanidades, es decir, discusiones críticas o deconstructivas, lo propio del hombre o los derechos humanos que tienen su espacio legítimo "en la universidad y, dentro de ella, con especial relevancia, en las Humanidades" (Derrida, 2002: 11) no con el objetivo de encerrarse dentro de ellas, sino -por el contrario- "para encontrar el mejor acceso a un nuevo espacio público transformado por unas nuevas técnicas de comunicación, de información, de archivación y de producción de saber" (Íbid. 12). Por tal motivo, propone ampliar y reelaborar el concepto de Humanidades, sin dejar de lado los antiguos cánones, pero incluir unas "Humanidades transformadas" (Íbid. 20). Derrida afirma que estas nuevas humanidades se desarrollan en un nuevo espacio, el de la virtualización, que afecta al lugar y a la naturaleza del trabajo universitario y desestabiliza el hábitat universitario.

Dentro de la Web hay espacio a todo tipo de expresiones culturales, artísticas o sociales. La cibercultura es híbrida en todo sentido, pues se funden y se mezclan los mass media, los distintos tipos de textos y las diversas formas de comunicación. El ciberespacio no es propiedad de nadie: es propiedad de todos y para comprender la cibercultura (De Kerckhove, 1997) es necesario hacerlo desde tres grandes características: la interactividad, la hipertextualidad y la conectividad, o Webness ${ }^{23}$. El resultado de las tres características promueven lo que él denomina las inteligencias en conexión, es decir, "el incremento en las interacciones humanas -personales, sociales e institucionales-, a través de las redes integradas, está concentrando y multiplicando la energía mental humana" (De Kerckhove, 1999: 175) y, desde esta perspectiva, las humanidades pueden potenciarse en el ambiente digital. Como afirman Burdick, Drucker, Lunenfeld, Presner y Schnapp (2012), modelar el conocimiento en entornos digitales requiere las perspectivas de humanistas, diseñadores y tecnólogos.

Las fuentes electrónicas constituyen uno de los más importantes recursos con que cuentan los investigadores, ya que permiten la consulta de trabajos recientemente editados o bien el acceso a material anteriormente restringido

\footnotetext{
${ }^{23}$ Para Kerckhove, "Es la convergencia de hipertexto, multimedia, realidad virtual, redes neuronales y vida artificial que configuran una nueva condición cognitiva denominada Webness, es decir, la esencia de toda red..
} 
$\mathrm{y}$, de hecho, las instituciones de educación superior ponen al alcance de la comunidad universitaria las bases de datos electrónicas que permiten acceder a las fuentes bibliográficas requeridas en la actividad académica. McLeod, Broady-Preston y Atkins (2014) afirman que actualmente en la mayoría de las bibliotecas se lleva a cabo alguna forma de digitalización, rasgo que se constituye como la base de la investigación en humanidades.

La tecnología de la información está creando cambios masivos entre la comunidad académica con relación a cómo se trabaja y se comunican los resultados de las investigaciones, cómo producen los estudiantes y cómo se enseña en las universidades. Las formas tradicionales de comunicación en ciencias están siendo transformadas por el uso de las tecnologías de la información, particularmente en las etapas de edición y distribución del conocimiento (Patalano, 2005: 218).

En otras palabras, la digitalización no debe ser entendida como la antítesis de las humanidades, pues -como afirma Bell (1973) la sociedad postindustrial se caracteriza por la informática, el uso del computador y los sistemas de transmisión de datos como medios de transformación, el saber como medio estratégico, la tecnología del intelecto como tecnología y la codificación del saber teórico como principio fundamental (Bell, 1981: 54). Lo planteado permite comprender una nueva perspectiva desde la cual es posible compatibilizar humanidades y tecnología: las humanidades digitales, concepto que ha ido enriqueciéndose al expandirse su uso y que remiten a una "humanización" de la tecnología. En un principio se utilizó para referirse a los recursos electrónicos, especialmente los procesos de digitalización de documentos impresos, pero luego se añadió el análisis del impacto de las tecnologías informáticas en estas disciplinas, como también el proceso de replantear el trabajo humanístico en la sociedad de la información, lo cual implica un enfoque teórico y filosófico propios (Leibrandt, 2006). Para Jänicke, Franzini et al. (2015) el cambio de leer un solo libro "en papel” a la posibilidad de navegar por muchos textos digitales es uno de los orígenes y pilares principales del dominio de las humanidades digitales. Los científicos de informática y los estudiosos de las humanidades aparentemente no tienen muchas cosas en común. Aunque comparten algunas metodologías, están 
orientadas hacia diferentes objetivos, pero la era digital creó una plataforma que reúne a personas de dos áreas de investigación: las humanidades digitales.

Para Galina (2011), los objetivos de las humanidades digitales se sintetizan en tres ejes: 1) Crear bases de datos con recursos digitales relevantes para las humanidades, lo cual incluye la captura, estructuración, documentación, preservación y diseminación de los datos; 2) Desarrollar metodologías que permitan generar nuevos elementos derivados de estos datos y 3) Generar investigación y conocimiento para incrementar nuestra comprensión en las humanidades. Marín Dacos (2011) sostiene que los profundos cambios experimentados en nuestra sociedad en el ámbito digital modifican y cuestionan las condiciones de producción de los saberes, por lo cual las humanidades digitales abarcan el conjunto de las Ciencias Humanas y Sociales, de las Artes y de las Letras. Pero no reniegan del pasado, sino que se apoyan en el conjunto de los paradigmas, saberes y conocimientos propios de esas disciplinas a la par que movilizan herramientas y perspectivas propias del campo digital. De acuerdo con Digital Humanities Manifesto 2.0 (2009), las humanidades digitales pretenden desempeñar un papel inaugural en un mundo en el que las universidades, si bien ya no son las únicas productoras, administradoras y divulgadoras de conocimientos o de la cultura, están llamadas a dar forma a los modelos digitales del discurso académico para las emergentes esferas públicas de la época actual (la WWW, las bibliotecas digitales, etc.) para modelar la excelencia y la innovación en estos ámbitos, y para facilitar la formación de redes de producción de conocimiento, intercambio y difusión tanto global como local. Se aprovechan las herramientas digitales al servicio de las fortalezas metodológicas básicas de las humanidades: la atención a la complejidad, el contexto histórico, la profundidad analítica, la crítica y la interpretación. Según Katz (2005), la asociación creativa entre la informática y las ciencias humanas -las llamadas "humanidades digitales" - es la piedra angular de la revolución digital. Por este motivo, según Davidson (2008, en Conway 2010), tal vez se deben ver la tecnología y las humanidades no como contrapuestas, sino como las dos caras de un conjunto necesariamente interdependiente, unidas y mutuamente constitutivas de prácticas intelectuales, 
educativas, sociales, políticas y económicas.

\section{Conclusión}

Byung-Chul Han (2013) sostiene que hoy nos encontramos en una nueva crisis, "en una transición crítica, de la cual parece ser responsable otra transformación radical: la revolución digital" (Byung, 2014: 15). En un mundo digitalizado, las bases de datos, las enciclopedias electrónicas, la digitalización de documentos o la reconstrucción virtual de espacios históricos son todas muestras de que es posible compatibilizar humanidades, ciencia y tecnología, tal como lo plantean las humanidades digitales.

Asimismo, las humanidades y las ciencias no se oponen, sino que se complementan. Los dos enfoques obedecen al deseo inherente del hombre por conocer y explicar no solo cuanto lo rodea, sino también por la creación o la reflexión respecto de quiénes somos. Para Small (2013), las humanidades pueden tener valor de cinco maneras primarias: por su comprensión del significado y el conocimiento; su distancia de las aplicaciones prácticas ${ }^{24}$; su contribución a la felicidad ${ }^{25}$; su contribución a la democracia; por su propio bien. La demanda final es que las humanidades importan por sí mismas.

A diferencia del discurso de la ciencia, el de las humanidades es un discurso de permanencia que nos ancla a la tradición cultural, a los saberes que no se desechan, sino que se articulan con otros. Pero literatura, arte, historia o filosofía no solo miran al pasado, pues continuamente se van renovando y cada generación aporta nuevos saberes a los ya existentes. La tecnología digital puede ser una poderosísima herramienta que no solo permite preservar dichos saberes, sino que también ser una impulsora de nuevos conocimientos significativos y profundos, propios de la humanidad, por lo cual quienes

\footnotetext{
24 "There is an old line of argument that the humanities are necessarily (some will say laudably) useless, or at a remove from accounts of practical ends and economic utility".

25 "The core assertion here is not the (overpitched) one that "the humanities will make you happy'; rather, that the humanities can help us to understand better what happiness is, how we may better put ourselves in the way of it, and how education may improve the kind and quality of some of our pleasures".
} 
estudiamos literatura, lenguaje, artes, filosofía no debemos abandonar el interés por seguir colaborando con el saber y la cultura desde las humanidades.

\section{Referencias}

Albarello, F. (2011). Leer/navegar en Internet. Las formas de lectura en la computadora. Buenos Aires: La Crujía Ediciones.

Arbea, A. (2002). El concepto de Humanitas en el Pro Archia de Cicerón. Onomazein 7, 393-400.

Ardèvol, E. (2002). “Cibercultura / cibercultures: La cultura d'Internet o l'anàlisi cultural dels usos socials d'Internet". IX Congrés d'Antropologia FAAEE, Barcelona. [en línea]. Disponible en: http:// www.academia.edu/704888/Cibercultura_ciberculturas_La_cultura de_Internet_o_el_an\%C3\%A1lisis_cultural_de_los_usos_sociales de_Internet (Consultado el 15 junio de 2016).

Belfiore, E.; Upchurch, A. R. (2013). Humanities in the Twenty-First Century. Beyond Utility and Markets. New York: Palgrave and Mcmillan.

Bérube, M. (2017) "Why Fund Humanities Research?” [en línea]. Disponible en: https://chcinetwork.org/ideas/the-humanities-and-theadvancement-of-knowledge (Consultado el 20 de mayo de 2018).

Bod, R. (2013) A new History of Humanities. The Search for Principles and Patterns from Antiquity to the Present. Oxford: Oxford University Press.

Brockman, J. (1995). The Third Culture. Beyond the Scientific Revolution. New York: Simon \& Schuster. 
Burdick, A.; Drucker, J.; Lunenfeld, Peter; P., Todd; Schnapp, J. (2006). "Humanities to Digital Humanities". Digital Humanitis, Cambridge, Massachusset: The MIT Press.

Busl, G. (2015). "Humanities research is groundbreaking, life-changing... and ingnored". [en línea]. Disponible en: https://www.theguardian. com/higher-education-network/2015/oct/19/humanities-research-isgroundbreaking-life-changing-and-ignored (Consultado el 15 de junio de 2018).

Byung-Chul H. (2014). En el enjambre. Barcelona: Herder.

Castells, M. (2006). La Era de la Información: Economía, sociedad y cultura, Volumen I: La Sociedad Red, México: Siglo XXI Editores.

Cordua, C. (2012). "La crisis de las humanidades". Revista de Filosofía, Vol. $68,7-9$.

Cordua, C. (2013). "El Humanismo". Revista Chilena de Literatura $\mathrm{N}^{\circ} 84$, 9-17.

De Kerckhove, D. (1997). The skin of culture: investigating the new electronic reality, London: Kogan Page Limited.

De Kerckhove, D. (1999) Inteligencias en conexión. Hacia una sociedad de la Web. Barcelona: Gedisa.

De Zubiría, R. (1990). “Acerca del concepto de las humanidades”, Thesaurus, Tomo XLV, N², 489-496.

Derrida, J. (2002). La universidad sin condición. Madrid: Ed. Trotta. 
Dery, M. (1998. Velocidad de escape. La cibercultura en el final del siglo, Madrid: Siruela.

Diéguez Lucena, A. (2000). “¿Hubo siempre dos culturas?”. Contrastes. Revista interdisciplinar de Filosofia, Vol. V, 43-60.

European Comission. "Social Sciences and Humanities". Horizon 2020. The EU Framework Programme for Research and Innovation (2017) [en línea]. Disponible en: https://ec.europa.eu/programmes/horizon2020/ en/area/social-sciences-and-humanities (Consultado el 5 abril de 2018).

Gould, S. J. (2010). Érase una vez el zorro y el erizo. Las humanidades y la ciencia en el tercer milenio. Barcelona: Crítica.

Heidegger, M. (2010). Carta sobre el Humanismo. Madrid: Alianza.

Hutner, G. y M., Feisal G. (2016). A new deal for the humanities. Liberal arts and the future of public higher education. New Brunswick: Tutgers.

Jänicke, S.; Franzini, G.; Cheema, M. F.; Scheuermann, G. (2015). “On Close and Distant Reading in Digital Humanities: A Survey and Future Challenges". R. Borgo, F. Ganovelli; I. Viola (Eds.) Eurographics Conference on Visualization (EuroVis) STAR - State of The Art Report. [en línea]. Disponible en: https://pdfs.semanticscholar. org/20cd/40f3f17dc7d8f49d368c2efbc2e27b0f2b33.pdf (Consultado el 18 abril de 2018).

Joyanes, L. (1997). Cibersociedad, los retos sociales ante un nuevo mundo digital. Madrid: McGraw-Hill. 
Kagan, J. (2009). The Three Cultures. Natural Sciences, Social Sciences, and the Humanities in the 21st Century. Cambridge: Cambridge University Press.

Kaplan, I. (2017). “The Way We Judge Humanities Professors Is Broken-This Initiative Could Fix It" [en línea]. Disponible en: https://www.artsy. net/article/artsy-editorial-way-judge-humanities-professors-brokeninitiative (Consultado el 14 abril de 2018).

Leavis, F. R. (1962). "Two cultures?: The significance of C. P. Snow". Richmond Lecture. New York: Cambridge University Press.

Lévy, P. (2007). Cibercultura. La cultura de la sociedad digital. México: Ed. Anthropos.

Llopis Goig, R. (2008). "La tercera cultura de Brockman". Revista A distancia, V. 23, No 4, 169-175.

López-Bonilla, G. (2013). “Prácticas disciplinares, prácticas escolares. Qué son las disciplinas académicas y cómo se relacionan con la educación formal en las ciencias y en las humanidades". Revista Mexicana de Investigación Educativa, Vol. 18, N 57, 383-412.

Mann, N. (1998). "Orígenes del humanismo", en J. Kraye (Ed.) Introducción al humanismo renacentista. Madrid: Cambridge University Press, 1939.

McLeod, L.; Broady-Preston, J.; Atkins (2014). “Accessing Humanities Research in a Digital Environment". Procedia. Social and Behavioral Sciences 147, 140-146. 
Moosa, I. A. (2018). Publish or Perish: Perceived Benefits Versus Unintended Consequences. Cheltenham: Edward Elgar Publishing.

Morón Arroyo, C. (2002). The Humanities in the Age of Technology. The Catholic University of America Press.

Nussbaum, M.(2010). Sin fines de lucro. Por qué la democracia necesita de las humanidades. Buenos Ares: Katz Editores.

Patalano, M. (2005). "Las publicaciones del campo científico: las revistas académicas de América Latina". Anales de Documentación $\mathrm{N}^{\circ}$ 8, 217 235 .

Piscitelli, A. (2002). Ciberculturas 2.0. En la era de las máquinas inteligentes. Buenos Aires: Paidós.

Proctor, R. E. (1998). Defining the Humanities: How Rediscovering a Tradition Can Improve Our Schools. Bloomington: Indiana University Press.

Real Academia Española (2014). Diccionario de la Lengua Española. Madrid: Espasa-Calpe, $23^{a}$ edición.

Reale, E.; Avramov, D.; Canhial, K.; Donovan, C.; Flecha, R.; Holm, P.; Larkin, C.; Lepori, B.; Mosoni-Fried, J.; Oliver, E.; Primeri, E.; Puigvert, L.; Scharnhorst, A.; Schubert, A.; Soler, M.; Soòs, S.; Sordé, T.; Travis, C.; Van Horik, R. (2017) "A review of literature on evaluating the scientific, social and political impact of social sciences and humanities research". Research Evaluation, Special Issue, 1-11 [en línea]. Disponible en: https://academic.oup.com/rev/advance-article/doi/10.1093/reseval/ rvx025/3978693 (Consultado el 4 de abril de 2018). 
Reynal, V. (1995). Introducción a las humanidades. Río Piedras: Editorial de la Universidad de Puerto Rico.

Russell, B. (1997). My Philosophical Development, London: Routledge.

Santos Herceg, J. (2012). "Tiranía del paper. Imposición institucional de un tipo discursivo". Revista Chilena de Literatura No 82, 197-217.

Scolari, C. (2008). Hipermediaciones. Barcelona: Gedisa.

Small, H. H. (2013). The Value of the Humanities. Oxford: Oxford University Press.

Snow, C. P. (2000). Las dos culturas. Buenos Aires: Nueva Visión.

Sokal, A.; Bricmont, A. (1999). Imposturas intelectuales. Barcelona: Paidós.

Thelwall, M.; Delgado, M. M. (2015). "Arts and humanities research evaluation: no metrics please, just data". Journal of Documentation, Vol. 71, Iss. 4, 817-833.

Tofts, D. J.; Mckeich, M. (1998). Memory Trade: A Prehistory of Cyberculture. Newark: Gordon \& Breach Publishing Group. 